\title{
Improving the Fire Retardant Efficiency of Phosphazenes for some Constructional Materials
}

\author{
Hyder I. AL Taha \\ Asaad F. Khattab \\ Department of Chemistry/ College of Sciencel University of Mosul
}

(Received 14/3/2019; Accepted 15/4/2019)

\begin{abstract}
Cyclophosphazene and Polyphosphazene have been developed as a fire retarding material by increasing the number of bromine and chlorine atoms and adding azo moieties within the chemical structure. Four azo phenolic compounds with different numbers and places of substituted bromine and chlorine atoms were prepared. The cyclophosphazene and polyphosphazene were functionalized by the azo phenolic compounds. The functionalizing was done by substituting three chlorine atoms from cyclophosphazene or one chlorine atom from polyphosphazene by the prepared azo compounds through hydroxyl group. (ratio Azo3:1Cyclophosphazoene, and ration Azo1:1 Polyphosphazene). The chemical structure of the phosphazenes were studied by IR, ${ }^{1} \mathrm{H}-\mathrm{NMR}$ and elemental analysis. The fire retarding efficiency of phosphazenes compounds for many trading constructional materials was examined. The efficiency of the new phosphazenes compounds was increased for both Cyclophosphazene and Polyphosphazene as a fire retardant.
\end{abstract}

Keywords: phosphazenes, polyphosphazene, azo compound, fire retardant.
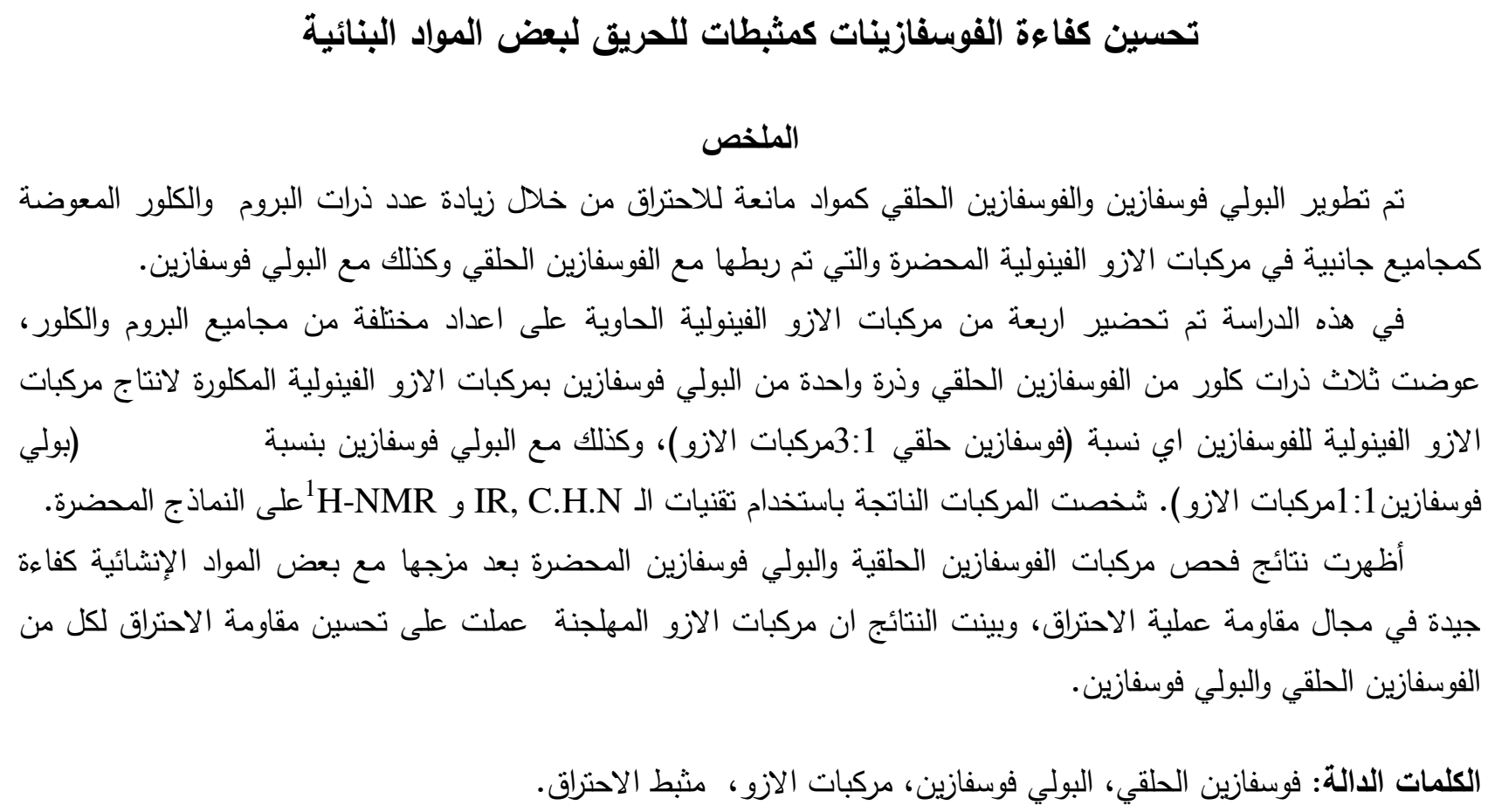
Fire retardancy of the composite materials can be achieved by inhibiting or by disrupting the continuous combustion process. Combustion process for composite materials can be interrupted in three different ways (Dittenber and Gangarao, 2012).

Firstly, by incorporating fire retardant materials that decompose endothermically when exposed to reach the pyrolysis temperature of composite materials (Camino and Cortemiglia, 1991).

Secondly, by incorporating fire retardant that produce more non-flammable byproducts and more char during pyrolysis reaction. The char layer acts as a physical barrier which hinders the heat and mass transfer between gas and condense phase. This mechanism is known as condensed phase mechanism (Camino and Cortemiglia, 1991).

Third, method is known as gas phase mechanism, in this case during combustion, the fire retardant chemicals release more non-flammable gases leading to reduction the an effective oxygen concentration in the flame zone and thus act as a fire retardant-agent (Schindler and Hauser, 2004).

Fire retardants behavior or fire performance of a composite materials can be improved in two ways. Firstly, by improving the fire performance of composite making constituents i.e. matrix and reinforcing agent. Secondly, by providing protective fire retardant coating around the core composites (Wichman, 2003). Different methods of improving fire performance of composite materials have been done through component modification as well as through providing protective coating. (Cullis and Hirschter, 1981).

Polymeric composite materials are widely used in different areas where fire retardancy one of the most important requirements. It is observed that fire retardant treatment not only affect the fire performance of composite materials it also affects the other composite properties like mechanical or physical properties (Shumao et al., 2010).

\section{Materials and Equipments:}

\section{EXPERIMENTAL}

All chemical and reagents are purchased from Fluka and used as received without purification. The used constructional materials are shown in (Table 1).

Table 1: Construction materials Source

\begin{tabular}{|c|c|c|}
\hline No. Sample & Commercial Materials & Source \\
\hline 1 & Epoxy (Ep) & CALIDAD HENKEL in Greece \\
\hline 2 & X-SBR & Durmakker.Evcrgem. in Belgium \\
\hline 3 & Co polystyrene (AS) & Durmakker.Evcrgem. in Belgium \\
\hline 4 & polyurethane primer(PU) & ALchimica Builing Chemicals in Greece \\
\hline 5 & polyurethane foam(PU.F) & ALchimica Builing Chemicals in Greece \\
\hline 6 & PVA (Polyvinyl acetate) & Shenxhen Taiqiang Chemicals in Chine \\
\hline 7 & Pint(OD) & Local \\
\hline 8 & Wood "saw dust"(W) & Local \\
\hline
\end{tabular}

${ }^{1} \mathrm{H}$ NMR(400 MHz) spectra were obtained by using (Bruker BioSpin GmbH in turkey). Tetramethyl saline was used as reference. FTIR spectra were accomplished by using (BRUKER FTIR Infrared Spectrophotometer).

The elemental analysis were carried out by using ( EuroEA3000/Italy) elemental a analyzer.

\section{Synthetic Route}


Azo compounds were prepared (Table 2) according to the following general method: (Khattab and Abbas, 2015) 0.01 mole of aniline or its derivatives were dissolved in $10 \mathrm{ml}$ of concentrated hydrochloric acid at $\left(-10^{\circ} \mathrm{C}\right)$. An aqueous solution of $0.01 \mathrm{M}$ of sodium nitride was added drop wise with stirring at $\left(-10^{\circ} \mathrm{C}\right)$ for a period of 30 minutes. Phenol compound $(0.01$ mole $)$ was dissolved in $10 \mathrm{ml}$ of sodium hydroxide solution $(10 \%)$ and added to the suspended dizonium salt solution drop wise and stirred for $1.5 \mathrm{hr}$. The precipitated azo compound was filtered, washed with cold water and dried under vacuum.

Hexachlorocyclophosphazene $\left(\mathrm{NPCl}_{2}\right)_{3}$ was prepared by refluxing equimolar of phosphorous pentachloride with ammonium chloride in chlorobenzene for $8 \mathrm{hrs}$. The reaction mixture was filtered from unreacted ammonium chloride, and the filtrate was distilled under vacuum. The residue phosphazene was recrystallized from hexane (yield 26\%). Polyphosphazene $\left[\mathrm{NPCl}_{2}\right]_{\mathrm{n}}$ was prepared by heating the cyclophosphazene $\left(\mathrm{NPCl}_{2}\right)_{3}$ at $200{ }^{\circ} \mathrm{C}$ for 3 hour under vacuum. (Allcock, 2006).

Table 2: The chemical structure of azo compounds and their physical properties

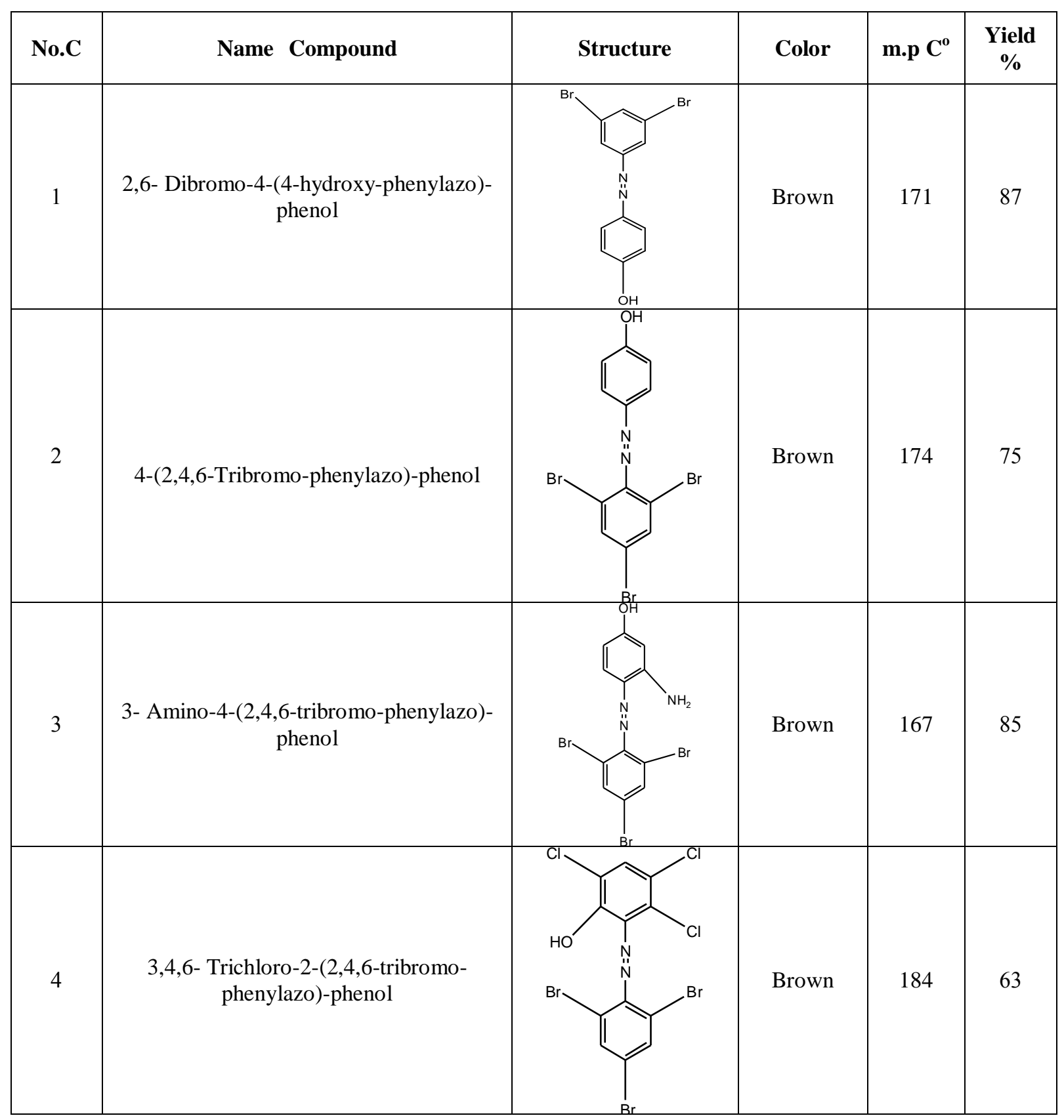


In a porcelain crucible 2 mmole of $\left(\mathrm{NPCl}_{2}\right)_{3}$ was fused by using a sand bath $\left(\right.$ at $\left.112{ }^{0} \mathrm{C}\right)$ followed by adding 6 mmole of azo compound. Heating the mixture was continued until vapor of hydrochloric acid was noticed (tested by litmus paper). The precipitate was cooled and stored in a vacuum dryer (Allcock 2012). (Table 3).

Table 3: The chemical structure of phosphazenes compounds (M) and some of its physical properties

\begin{tabular}{|c|c|c|c|c|}
\hline Samples & Compound Name & Chemical Stricture & $\begin{array}{c}\text { M.P } \\
\text { C }^{\mathbf{0}}\end{array}$ & $\begin{array}{c}\text { Yield } \\
\%\end{array}$ \\
\hline M1 & 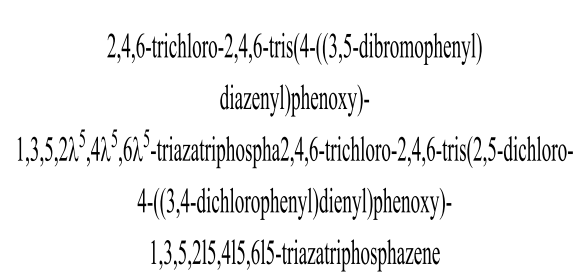 & & 204 & 74 \\
\hline M2 & $\begin{array}{c}\text { 2,4,6-trichloro-2,4,6-tris(4- } \\
\text { ((2,4,6-tribromophenyl)diazenyl)phenoxy)- } \\
1,3,5,2 \lambda^{5}, 4 \lambda^{5}, 6 \lambda^{5} \text {-triazatriphosphinine }\end{array}$ & & 171 & 89 \\
\hline M3 & $\begin{array}{l}\text { 5,5',5"-((2,4,6-trichloro-1,3,5,2, } \lambda^{5}, 4 \lambda^{5}, 6 \lambda^{5}- \\
\text { triazatriphosphinine-2,4,6-triyl)tris(oxy)) } \\
\text { tris(2-((2,4,6-tribromophenyl)diazenyl)aniline) }\end{array}$ & & 217 & 65 \\
\hline M4 & $\begin{array}{c}\text { 2,4,6-trichloro-2,4,6-tris(3,4,6-trichloro-2- } \\
\text { ((2,4,6-tribromophenyl)diazenyl)phenoxy)- } \\
1,3,5,2 \lambda^{5}, 4 \lambda^{5}, 6 \lambda^{5} \text {-triazatriphosphinine }\end{array}$ & & 174 & 56 \\
\hline
\end{tabular}

Functionalizing $\left[\mathrm{NPCl}_{2}\right]_{n}$ with Azo Compounds 
Dissolved $17.0 \mathrm{mmol}$ of Polyphosphazene $\left[\mathrm{NPCl}_{2}\right] \mathrm{n}$ in $50 \mathrm{ml}$ THF (Tetrahydrofuran) and dissolve $34.0 \mathrm{mmol}$ from azo compounds in $50 \mathrm{ml} \mathrm{THF}$, add $20 \mathrm{gm} \mathrm{K}_{2} \mathrm{CO}_{3}$. Refluxing the mixture for 9 hour and filtration the mixture to remove $\mathrm{KCl}$ salt, deposit polyphosphazene was obtained by using petroleum ether 60-80. (Arvind et al., 2010). (Table 4)

Table 4: Name and structure of the polyphosphazenes compounds (Pz)

\begin{tabular}{|c|c|c|}
\hline No. comp. & Name compounds & Structure compounds \\
\hline Pz:1 & $\begin{array}{l}\text { 4-((3,5-dibromophenyl)diazenyl)phenyl } \\
\text { N,P-dimethylphosphazene }\end{array}$ & \\
\hline Pz:2 & $\begin{array}{l}\text { 4-((2,4,6-tribromophenyl)diazenyl)phenyl } \\
\text { N,P-dimethylphosphazene }\end{array}$ & \\
\hline Pz:3 & $\begin{array}{l}\text { 3-amino-4-((2,4,6-tribromophenyl)diazenyl)phenyl } \\
N, P \text {-dimethylphosphazene }\end{array}$ & \\
\hline Pz:4 & $\begin{array}{c}\text { 3,4,6-trichloro-2-((2,4,6-tribromophenyl)diazenyl)phenyl } \\
N, P \text {-dimethylphosphazene }\end{array}$ & \\
\hline
\end{tabular}


This test was accomplished according to method ASTM E 285-80 for all phosphazenes compounds with the some construction materials (Table 1) The mixing percentage was $1 \%$. The wood was prepared by curing the saw dust with resole in presence of phosphazenes compounds. The properties of fire retarding were calculated as following:

Insulation Index (I.I) was recorded according to the equation:

$\mathrm{I} . \mathrm{I}=\mathrm{B} . \mathrm{T}(\mathrm{sac}) / 4(\mathrm{~mm})$ where B.T = Burning Time $/ \mathrm{sec}, 4 \mathrm{~mm}=$ Sample thick

Erosion Rate (E.R) was calculated according to the equation:

E.R $=4 \mathrm{~mm} / \mathrm{B} \cdot \mathrm{T} \mathrm{sec}$

The Percentage of residual weight of combustion was calculated according to the equation:

$\mathrm{RWR}=(\mathrm{W} 1-\mathrm{W} 2 / \mathrm{W} 1) * 100$

Where $: \mathrm{W} 1=$ Sample weight before burning $(\mathrm{gm}), \mathrm{W} 2=$ Weight of the missing material $(\mathrm{gm})$.

\section{RESULTS AND DISCUSSION}

Preparation polyphosphazene and cyclophosphazene coupled with azo phenolic compounds is involved abbreviate and rapid method in comparing with the general method (Allcock, 2006). Method preparing cyclophosphazene with azo phenolic compounds involved fusing mixture of hexachlorocyclotriphosphazene $\left(\mathrm{NPCl}_{2}\right)_{3}$ with the phenolic compounds. Accordingly, $\left(\mathrm{NPCl}_{2}\right)_{3}$ was treated with three molar equivalents of phenolic compounds (Table 2) to yield triphenyl diazenyl phenoxy phosphazene (Table 3).

The reaction of polyphosphazene with azo phenolic compounds was carried out by refluxing involved dissolved polyphosphazene and azo phenolic compounds in THF in presence of $\mathrm{K}_{2} \mathrm{CO}_{3}$.

The prepared azo compounds is diagnosed by IR (Table 5), Fig. (1). The molecular structure of the resulted cyclophosphazenes were assessed by IR (Table 6), Fig. (2) and ${ }^{1}$ H-NMR (Table 7), Fig. (3) spectroscopy, as well as elemental analysis.

Table 5: F.T. IR band frequencies of the prepared azo phenolic compounds

\begin{tabular}{|c|c|c|c|c|c|}
\hline Compounds & $\begin{array}{c}\text { ט O-H } \\
\mathrm{Cm}^{-1}\end{array}$ & $\begin{array}{c}\text { v } \mathrm{N}=\mathrm{N} \\
\mathrm{Cm}^{-1}\end{array}$ & $\begin{array}{c}\text { ט } \mathrm{C}=\mathrm{C} \\
\mathrm{Cm}^{-1}\end{array}$ & $\begin{array}{c}\text { ט C-Br } \\
\mathrm{Cm}^{-1}\end{array}$ & $\begin{array}{r}\text { ט C-N } \\
\mathrm{Cm}^{-1}\end{array}$ \\
\hline 1 & 3247 & 1585 & $1225-1496$ & 483 & 1136 \\
\hline 2 & 3239 & 1592 & $1370-1243$ & 599 & 1144 \\
\hline 3 & 3186 & 1500 & $1602-1237$ & 467 & 1091 \\
\hline 4 & 3362 & 1591 & $1544-1201$ & $\begin{array}{c}444 \\
\text { ט C-Cl } \\
753\end{array}$ & 1108 \\
\hline
\end{tabular}

Table 6: F.T. IR band frequencies of the prepared cyclophosphazenes compounds (M)

\begin{tabular}{|c|c|c|c|c|c|}
\hline Compound & $\begin{array}{c}\text { U P-O-C } \\
\mathrm{Cm}^{-1}\end{array}$ & $\begin{array}{c}\text { ט } \mathrm{N}=\mathrm{N} \\
\mathrm{Cm}^{-1}\end{array}$ & $\begin{array}{c}\text { ט } \mathrm{C}=\mathrm{C} \\
\mathrm{Cm}^{-1}\end{array}$ & $\begin{array}{c}\text { v C-Br } \\
\mathrm{Cm}^{-1}\end{array}$ & $\begin{array}{c}\text { u C-N } \\
\mathrm{Cm}^{-1} \\
\end{array}$ \\
\hline M1 & 999 & 1585 & $1225-1496$ & 483 & 1136 \\
\hline M2 & 993 & 1592 & $1370-1243$ & 599 & 1144 \\
\hline M3 & 976 & 1500 & $1602-1237$ & 467 & 1091 \\
\hline M4 & 999 & 1591 & $1544-1201$ & $\begin{array}{c}444 \\
\text { v C-Cl } \\
753\end{array}$ & 1108 \\
\hline
\end{tabular}


Table 7: ${ }^{1} \mathrm{H}$-NMR bands of some prepared phosphazenes (M)

\begin{tabular}{|c|c|c|c|}
\hline No. $M$ & Chemical Structure & \multicolumn{2}{|c|}{ Frequency } \\
\hline M1 & & $\begin{array}{l}(1,2) \\
(3,4) \\
(5,6) \\
(7)\end{array}$ & $\begin{array}{l}7.41 \\
7.61 \\
7.63 \\
7.64\end{array}$ \\
\hline M2 & 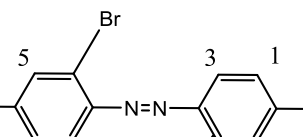 & $\begin{array}{l}(1,2) \\
(3,4) \\
(5,6)\end{array}$ & $\begin{array}{l}7.45 \\
7.65 \\
8.00\end{array}$ \\
\hline
\end{tabular}


Fig. 1: FTIR Spectra of azo compounds 

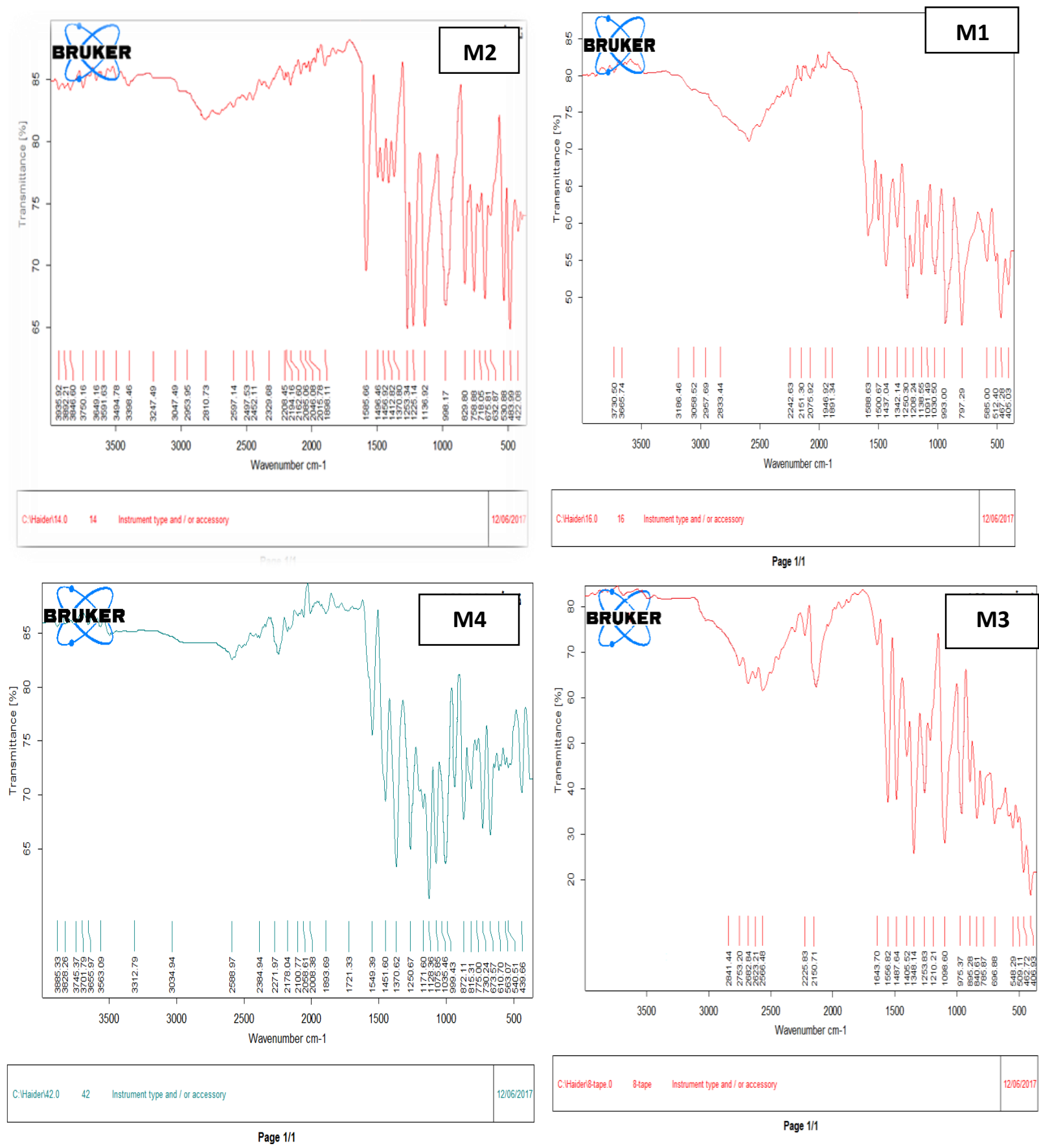

Fig. 2: FTIR Spectra of cyclophosphazenes compounds (M) 

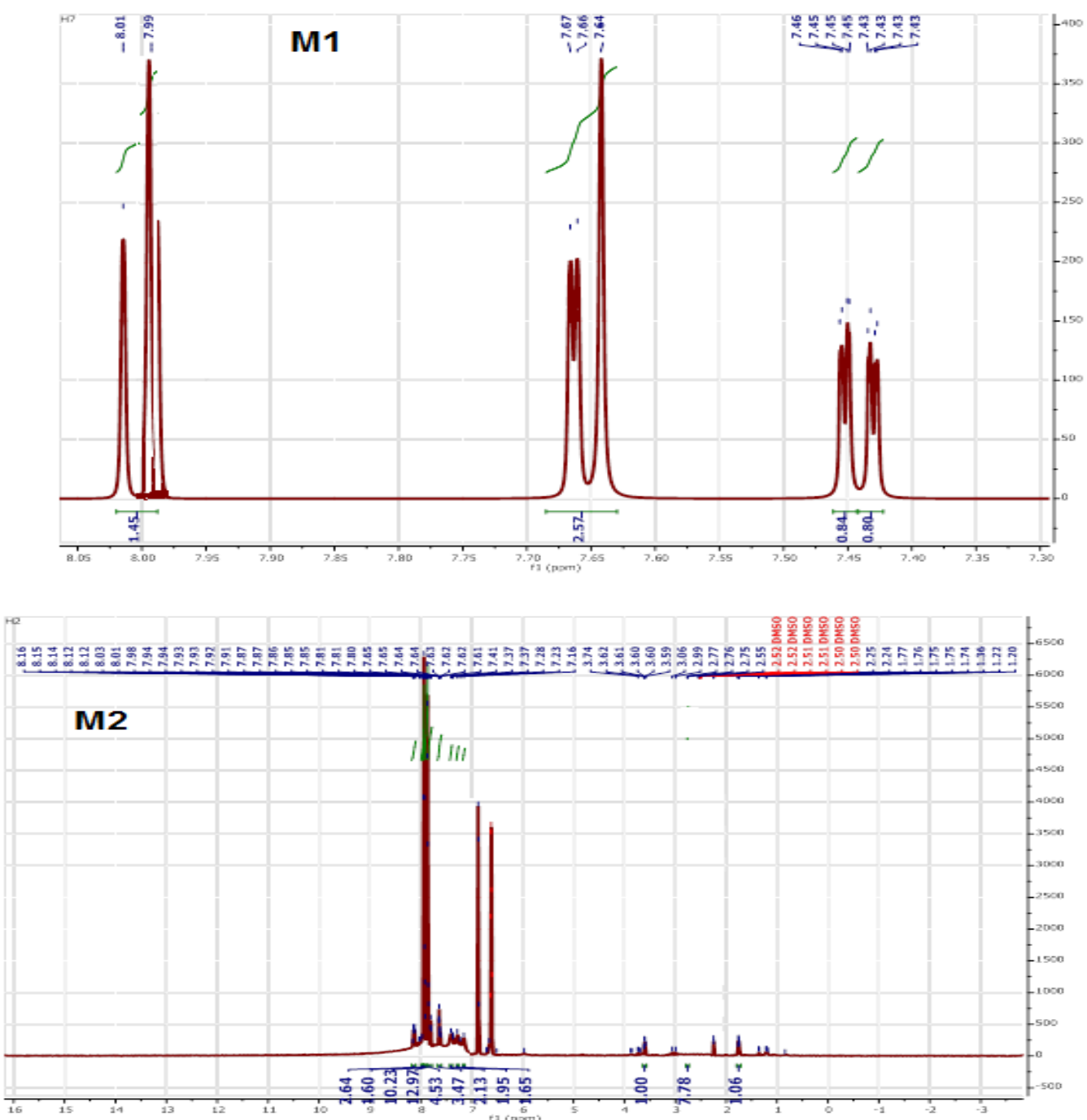

Fig. 3: ${ }^{1} \mathrm{H}-\mathrm{NMR}$ spectra of some prepared cyclophosphazenes compounds (M1, M2)

Polyphosphazenes were assessed by IR (Table 8), Fig. (4) and ${ }^{1}$ H-NMR (Table 9), Fig. (5) spectroscopy, as well as elemental analysis (Table 9). In comparison with the IR spectra of azo compounds, the spectra of the functionalized polyphosphazene and phosphazenes show the disappearance of $(\mathrm{OH})$ bands and appearance of new bands at about $1010-930 \mathrm{~cm}^{-1}$ for Ar-O-P, $1250-1150 \mathrm{~cm}^{-1}$ for $\operatorname{str}(\mathrm{P}=\mathrm{N})$ and $1256-1200 \mathrm{~cm}^{-1}$ for str. ${ }^{(11)}(\mathrm{P}-\mathrm{N})$ for all compounds.

These results presented the certainty that azo compounds were connected to cyclic phosphazene through (P-O-C) bonds.

Table 8: F.T. IR band frequencies of the prepared polyphosphazenes compounds $(\mathrm{Pz})$

\begin{tabular}{|c|c|c|c|c|c|}
\hline Compound & $\begin{array}{c}\text { U P-O-C } \\
\mathbf{C m}^{-1}\end{array}$ & $\begin{array}{c}\mathbf{0} \mathbf{~ N}=\mathbf{N} \\
\mathbf{C m}^{-1}\end{array}$ & $\begin{array}{c}\mathbf{U} \mathbf{C}=\mathbf{C} \\
\mathbf{C m}^{-1}\end{array}$ & $\begin{array}{c}\mathbf{0 ~ C - B r} \\
\mathbf{C m}^{-1}\end{array}$ & $\begin{array}{c}\mathbf{0} \mathbf{C}-\mathbf{N} \\
\mathbf{C m}^{-1}\end{array}$ \\
\hline Pz 1 & 999 & 1585 & $1225-1496$ & 483 & 1136 \\
\hline Pz 2 & 993 & 1592 & $1370-1243$ & 599 & 1144 \\
\hline Pz 3 & 976 & 1500 & $1602-1237$ & 467 & 1091 \\
\hline Pz 4 & 999 & 1591 & $1544-1201$ & $\begin{array}{c}444 \\
\mathbf{0 ~ C - C l} \\
753\end{array}$ & 1108 \\
\hline
\end{tabular}


Table 9: ${ }^{1} \mathrm{H}$-NMR bands of some prepared phosphazenes

\begin{tabular}{|c|c|c|c|}
\hline No. Pz & Chemical Structure & Frequ & ency \\
\hline $\mathrm{Pz} 2$ & & $\begin{array}{l}(1,3) \\
(2,4) \\
(5,6)\end{array}$ & $\begin{array}{l}2.99 \\
3.49 \\
7.88\end{array}$ \\
\hline Pz 4 & & $\begin{array}{l}(1) \\
(2,3)\end{array}$ & $\begin{array}{c}4.71 \\
7.49\end{array}$ \\
\hline
\end{tabular}


Fig. 5: ${ }^{1} \mathrm{H}-\mathrm{NMR}$ spectra of some prepared Polyphosphazenes compounds $(\mathrm{Pz})$

Elemental analysis of the functionalized cyclophosphazene and polyphosphazene, (Table 10), (Table 11) respectively, revealed certainly that the half amount of chlorine atom of $\left(\mathrm{NPCl}_{2}\right)_{3}$ and $\left[\mathrm{NPCl}_{2}\right]_{\mathrm{n}}$ were replaced by azo phenolic compounds. 
Table 10: Elemental analysis ( C.H.N) of cyclophosphazenes compounds(M)

\begin{tabular}{|c|c|c|c|c|c|c|}
\hline \multirow{2}{*}{ No. Sample } & \multicolumn{3}{|c|}{ \% Found } & \multicolumn{3}{c|}{ \% Calculated } \\
\cline { 2 - 7 } & \% N & \% H & \% C & \% N & \% H & \% C \\
\hline M1 & 13.029 & 2.989 & 33.997 & 13.524 & 2.986 & 33.500 \\
\hline M2 & 8.797 & 1.432 & 30.810 & 8.800 & 1.301 & 30.00 \\
\hline M3 & 10.974 & 1.491 & 27.116 & 10.518 & 1.389 & 26.856 \\
\hline M4 & 7.120 & 0.754 & 23.733 & 6.710 & 0.500 & 23.300 \\
\hline
\end{tabular}

Table 11: Elemental analysis ( C.H.N) of Polyphosphazenes compounds $(\mathbf{P z})$

\begin{tabular}{|c|c|c|c|c|c|c|}
\hline \multirow{2}{*}{ No . Sample } & \multicolumn{3}{|c|}{ \% Found } & \multicolumn{3}{c|}{ \% Calculated } \\
\cline { 2 - 7 } & \% N & \% H & \% C & \% N & \% H & \% C \\
\hline Pz 1 & 9.790 & 1.921 & 33.997 & 9.644 & 1.607 & 33.065 \\
\hline Pz 2 & 11.028 & 1.022 & 27.500 & 10.576 & 1.322 & 27.988 \\
\hline Pz 3 & 10.974 & 1.491 & 27.210 & 10.576 & 1.322 & 27.988 \\
\hline Pz 4 & 6.820 & 0.500 & 23.703 & 6.796 & 0.485 & 23.301 \\
\hline
\end{tabular}

\section{Flame Retarding Tests}

Construction materials have been mixed with $1 \%$ of fire retardant materials in order to improve their fire retarding characteristics. The homogeneity was rationalized via Atomic Force Microscopy (AFM) Fig. (6).



Fig. 6: AFM of mixing SBR with \%1 of phosphazene compound (M2)

Table (12) revealed the efficiency for fire retardant of the constructional materials with the prepared azo compounds. It was noticed very clear that increasing the number of halogen atoms will increased the efficiency of retarding the fire.

The efficiency was increased by many order of magnitude when their compounds are linked with the cyclotriphosphazene (Table 13). The result show also that cyclophosphazenes as a fire retardant was improved when its chemical structure was developed by the azo compounds whereas the number of halogen atoms have been increased.

The fluctuation in result is belong to the sites of substitution Polyphosphazenes compounds (Table 14) give a good satisfaction for fire retardency in comparison with the cyclophosphazenes or pure azo compounds. 
Table 12: The variables of fire retarding test for the construction materials composites with Azo compounds

\begin{tabular}{|c|c|c|c|c|c|c|c|c|c|c|c|}
\hline Material & Azo & $\begin{array}{l}\text { B.T/ } \\
\text { sec }\end{array}$ & I.I/ sec/mm & $\begin{array}{c}\text { E. R/ } \\
\mathrm{mm} / \mathrm{sec}\end{array}$ & $\begin{array}{l}\mathrm{H} / \\
\mathrm{cm}\end{array}$ & $\begin{array}{c}\mathrm{AEB} / \\
\mathrm{cm}\end{array}$ & $\begin{array}{l}\text { W1/ } \\
\text { gm }\end{array}$ & $\begin{array}{l}\text { W2/ } \\
\text { gm }\end{array}$ & $\begin{array}{c}\text { RWR } \\
\%\end{array}$ & E.S & N.B \\
\hline \multirow{5}{*}{ E.P } & Without & 46 & 11.5 & 0.086 & 5 & 8.5 & 5 & 2.00 & 60 & - & - \\
\hline & 1 & 48 & 12 & 0.083 & 5.0 & 8 & 5 & 1.80 & 64 & Yes & - \\
\hline & 2 & 33 & 8.25 & 0.121 & 4.0 & 5.5 & 5 & 1.29 & 74 & Yes & - \\
\hline & 3 & 27 & 6.75 & 0.148 & 4.0 & 4.5 & 5 & 1.00 & 80 & Yes & - \\
\hline & 4 & 28 & 7 & 0.142 & 3.5 & 5 & 5 & 1.09 & 78 & Yes & - \\
\hline \multirow{5}{*}{ P.U } & Without & 299 & 74.75 & 0.013 & 6.0 & 15 & 5 & 4.5 & 10 & - & - \\
\hline & 1 & 294 & 73.5 & 0.013 & 5.7 & 15 & 5 & 4.3 & 14 & - & - \\
\hline & 2 & 277 & 69.25 & 0.014 & 5.0 & 13 & 5 & 3.8 & 24 & - & - \\
\hline & 3 & 267 & 66.75 & 0.014 & 4.0 & 13 & 5 & 3.0 & 40 & - & - \\
\hline & 4 & 216 & 54 & 0.018 & 4.5 & 10 & 5 & 3.3 & 34 & - & - \\
\hline \multirow{5}{*}{ SBR } & Without & 575 & 143.75 & 0.006 & 12 & 15 & 5 & 4.6 & 8 & - & - \\
\hline & 1 & 542 & 135.5 & 0.007 & 11.5 & 14.8 & 5 & 4.4 & 12 & - & - \\
\hline & 2 & 451 & 112.75 & 0.008 & 10.6 & 11.4 & 5 & 3.8 & 24 & - & - \\
\hline & 3 & 446 & 111.5 & 0.008 & 9.5 & 8.7 & 5 & 3.1 & 38 & - & - \\
\hline & 4 & 441 & 110.25 & 0.009 & 9.6 & 9.4 & 5 & 3.3 & 34 & - & - \\
\hline \multirow{5}{*}{ A.S } & Without & 498 & 124.5 & 0.008 & 10.0 & 15.0 & 6 & 5.1 & 15 & - & - \\
\hline & 1 & 463 & 115.75 & 0.008 & 9.0 & 15 & 6 & 4.6 & 23 & - & - \\
\hline & 2 & 328 & 82 & 0.012 & 6.5 & 10.5 & 6 & 3.3 & 45 & - & - \\
\hline & 3 & 331 & 82.75 & 0.012 & 7.0 & 10.8 & 6 & 3.4 & 43 & - & - \\
\hline & 4 & 320 & 80 & 0.012 & 6.5 & 10.6 & 6 & 3.3 & 45 & - & - \\
\hline \multirow{5}{*}{ P.U.F } & Without & 306 & 76.5 & 0.013 & 12 & 15 & 4 & 3.6 & 10 & - & - \\
\hline & 1 & 295 & 73.75 & 0.014 & 11.4 & 14.5 & 4 & 3.4 & 15 & - & \\
\hline & 2 & 271 & 67.75 & 0.014 & 10.6 & 13.3 & 4 & 3.1 & 22 & - & - \\
\hline & 3 & 283 & 70.75 & 0.014 & 11.2 & 14.0 & 4 & 3.3 & 17 & - & - \\
\hline & 4 & 277 & 69.25 & 0.014 & 10.8 & 13.5 & 4 & 3.4 & 20 & - & - \\
\hline \multirow{5}{*}{ OD } & Without & 362 & 90.5 & 0.011 & 8.0 & 15 & 6 & 5.3 & 10 & - & - \\
\hline & 1 & 354 & 88.5 & 0.011 & 8.0 & 14.6 & 6 & 4.2 & 30 & - & - \\
\hline & 2 & 278 & 69.5 & 0.014 & 6.0 & 11.6 & 6 & 3.3 & 45 & - & - \\
\hline & 3 & 281 & 70.25 & 0.014 & 6.5 & 11.5 & 6 & 3.4 & 5343 & - & - \\
\hline & 4 & 285 & 71.25 & 0.0140 & 6.0 & 10.8 & 6 & 3.4 & 43 & - & - \\
\hline \multirow{5}{*}{ W } & Without & 588 & 147 & 0.007 & 12.0 & 15 & 5 & 4.8 & 4 & - & - \\
\hline & 1 & 510 & 127.5 & 0.0078 & 10.5 & 13.7 & 5 & 4.3 & 14 & - & - \\
\hline & 2 & 396 & 99 & 0.0101 & 8.0 & 10.0 & 5 & 4.1 & 18 & - & - \\
\hline & 3 & 426 & 106.5 & 0.0093 & 8.5 & 10.5 & 5 & 3.4 & 32 & - & - \\
\hline & 4 & 473 & 118.25 & 0.008 & 9.5 & 2.5 & 5 & 4.1 & 18 & - & - \\
\hline \multirow{5}{*}{ PVA } & Without & 372 & 93 & 0.011 & 13.0 & 15 & 6 & 4.5 & 25 & - & - \\
\hline & 1 & 368 & 92 & 0.011 & 13.0 & 15.0 & 6 & 4.5 & 25 & - & - \\
\hline & 2 & 338 & 84.5 & 0.012 & 12.0 & 14.6 & 6 & 4.0 & 33 & - & - \\
\hline & 3 & 341 & 85.25 & 0.012 & 12.0 & 4.4 & 6 & 4.3 & 28 & - & - \\
\hline & 4 & 329 & 82.25 & 0.012 & 11.5 & 14.2 & 6 & 3.9 & 35 & - & - \\
\hline
\end{tabular}


Table 13: The variables of fire retarding test for the construction materials composites with phosphazenes (M)

\begin{tabular}{|c|c|c|c|c|c|c|c|c|c|c|c|}
\hline Material & $\mathbf{M}$ & $\begin{array}{l}\text { B.T } \\
\text { sec }\end{array}$ & $\begin{array}{c}\text { I.I } \\
\text { sec/mm }\end{array}$ & $\begin{array}{c}\text { E.R } \\
\mathrm{mm} / \mathrm{sec}\end{array}$ & $\begin{array}{c}\mathbf{H} \\
\mathbf{c m}\end{array}$ & $\begin{array}{c}\text { AEB } \\
\text { Cm }\end{array}$ & $\begin{array}{l}\text { W1 } \\
\text { gm }\end{array}$ & $\begin{array}{l}\text { W2 } \\
\text { gm }\end{array}$ & $\begin{array}{c}\text { RWR } \\
\%\end{array}$ & E.S & N.B \\
\hline \multirow{6}{*}{ E.P } & Without & 46 & 11.5 & 0.086 & 5.0 & 8.5 & 5 & 2.0 & 60 & YES & - \\
\hline & $\mathrm{P}^{*}$ & 32 & 8 & 0.125 & 3.5 & 5.8 & 5 & 1.3 & 74 & YES & \\
\hline & M1 & 36 & 9 & 0.111 & 4.0 & 6.5 & 5 & 1.4 & 72 & YES & - \\
\hline & M2 & 23 & 5.75 & 0.173 & 2.5 & 4.0 & 5 & 0.9 & 82 & YES & - \\
\hline & M3 & 8 & 2 & 0.5 & 1.0 & 1.5 & 5 & 0.3 & 94 & YES & - \\
\hline & M4 & 4 & 1 & 1 & 0.5 & 1.0 & 5 & 0.1 & 98 & YES & - \\
\hline \multirow{6}{*}{ P.U } & Without & 299 & 74.75 & 0.013 & 6.0 & 15.0 & 5 & 4.5 & 10 & - & - \\
\hline & $\mathrm{P}^{*}$ & 162 & 40.5 & 0.025 & 3.0 & 8.0 & 5 & 2.4 & 52 & Yes & - \\
\hline & M1 & 168 & 42 & 0.023 & 3.5 & 8.4 & 5 & 2.5 & 50 & Yes & - \\
\hline & M2 & 153 & 38.25 & 0.026 & 3.0 & 7.6 & 5 & 2.3 & 54 & Yes & - \\
\hline & M3 & 44 & 11 & 0.090 & 1.0 & 2.2 & 5 & 0.7 & 86 & Yes & - \\
\hline & M4 & 29 & 7.25 & 0.137 & 0.5 & 1.5 & 5 & 0.4 & 92 & Yes & - \\
\hline \multirow{6}{*}{ SBR } & Without & 575 & 143.75 & 0.006 & 12 & 15 & 5 & 4.6 & 8 & - & - \\
\hline & $\mathrm{P}^{*}$ & 284 & 72 & 0.013 & 5.5 & 7.6 & 5 & 2.5 & 50 & Yes & - \\
\hline & M1 & 398 & 99.5 & 0.010 & 8.5 & 10.4 & 5 & 3.2 & 36 & - & - \\
\hline & M2 & 317 & 79.25 & 0.012 & 6.5 & 8.3 & 5 & 2.5 & 50 & Yes & - \\
\hline & M3 & 97 & 24.25 & 0.041 & 2.0 & 2.5 & 5 & 0.8 & 84 & Yes & - \\
\hline & M4 & 83 & 20.75 & 0.048 & 2.0 & 2.2 & 5 & 0.6 & 88 & Yes & - \\
\hline \multirow{6}{*}{ A.S } & Without & 498 & 124.5 & 0.008 & 10.0 & 15 & 6 & 5.1 & 15 & - & - \\
\hline & $\mathrm{P}^{*}$ & 182 & 45.5 & 0.022 & 3.5 & 5.3 & 6 & 1.7 & 71 & Yes & - \\
\hline & M1 & 302 & 75.5 & 0.013 & 6.0 & 9.2 & 6 & 3.0 & 50 & Yes & - \\
\hline & M2 & 164 & 41 & 0.024 & 3.5 & 5.0 & 6 & 1.6 & 73 & Yes & - \\
\hline & M3 & 126 & 31.5 & 0.031 & 2.5 & 3.8 & 6 & 1.3 & 78 & Yes & - \\
\hline & M4 & 21 & 5.25 & 0.190 & 0.5 & 0.6 & 6 & 0.2 & 95 & Yes & - \\
\hline \multirow{6}{*}{ P.U.F } & Without & 306 & 76.5 & 0.013 & 12 & 15 & 4 & 3.6 & 10 & - & - \\
\hline & $\mathrm{P}^{*}$ & 147 & 36.75 & 0.027 & 5.5 & 6.8 & 4 & 2.0 & 50 & Yes & - \\
\hline & M1 & 128 & 32 & 0.031 & 5.0 & 6.3 & 4 & 1.5 & 62 & Yes & - \\
\hline & M2 & 118 & 29.5 & 0.033 & 4.5 & 6.0 & 4 & 1.4 & 65 & Yes & - \\
\hline & M3 & 63 & 15.75 & 0.063 & 2.5 & 3.1 & 4 & 0.7 & 82 & Yes & - \\
\hline & M4 & 30 & 7.5 & 0.133 & 1.5 & 1.5 & 4 & 0.4 & 90 & Yes & - \\
\hline \multirow{6}{*}{ OD } & Without & 362 & 90.5 & 0.011 & 8.0 & 15 & 6 & 4.3 & 28 & - & - \\
\hline & $\mathrm{P}^{*}$ & 163 & 40.75 & 0.025 & 4.0 & 7.0 & 6 & 2.1 & 65 & Yes & - \\
\hline & M1 & 204 & 51 & 0.019 & 4.5 & 8.5 & 6 & 2.2 & 63 & Yes & - \\
\hline & M2 & 156 & 39 & 0.025 & 3.5 & 6.5 & 6 & 1.7 & 71 & Yes & - \\
\hline & M3 & 113 & 28.25 & 0.035 & 2.5 & 4.6 & 6 & 1.2 & 80 & Yes & - \\
\hline & M4 & 20 & 5 & 0.2 & 0.5 & 0.8 & 6 & 0.2 & 96 & Yes & - \\
\hline \multirow{6}{*}{ W } & Without & 588 & 147 & 0.006 & 12.0 & 15 & 5 & 4.8 & 4 & - & - \\
\hline & $* \mathrm{P}$ & 298 & 74.5 & 0.013 & 6.0 & 6.4 & 5 & 2.4 & 53 & - & - \\
\hline & M1 & 355 & 88.75 & 0.011 & 7.5 & 9.0 & 5 & 2.8 & 44 & - & - \\
\hline & M2 & 312 & 78 & 0.012 & 6.5 & 6.3 & 5 & 2.5 & 50 & Yes & - \\
\hline & M3 & 66 & 16.5 & 0.060 & 1.5 & 1.6 & 5 & 0.5 & 90 & Yes & - \\
\hline & M4 & 14 & 3.5 & 0.285 & 0.5 & 0.4 & 5 & 0.1 & 98 & Yes & - \\
\hline \multirow{6}{*}{ PVA } & Without & 372 & 93 & 0.010 & 13 & 15 & 6 & 4.5 & 25 & - & - \\
\hline & $* \mathrm{P}$ & 192 & 48 & 0.021 & 6.5 & 8.0 & 6 & 2.5 & 50 & Yes & - \\
\hline & M1 & 207 & 51.75 & 0.019 & 7.0 & 8.3 & 6 & 2.5 & 58 & Yes & - \\
\hline & M2 & 174 & 43.5 & 0.022 & 6.0 & 7.0 & 6 & 2.1 & 65 & Yes & - \\
\hline & M3 & 54 & 13.5 & 0.074 & 2.0 & 2.2 & 6 & 0.7 & 88 & Yes & - \\
\hline & M4 & 7 & 1.75 & 0.571 & 0.5 & 0.3 & 6 & 0.1 & 98 & Yes & - \\
\hline
\end{tabular}


Table 14: The variables of fire retarding test for the construction materials composites with Polyphosphazenes compounds (Pz)

\begin{tabular}{|c|c|c|c|c|c|c|c|c|c|c|c|}
\hline $\begin{array}{c}\text { Materi } \\
\text { al }\end{array}$ & $\mathbf{P z}$ & $\begin{array}{l}\text { B.T/ } \\
\mathrm{sec}\end{array}$ & $\begin{array}{c}\text { I.I/ } \\
\text { sec/mm }\end{array}$ & $\begin{array}{c}\text { E.R/ } \\
\mathrm{mm} / \mathrm{sec}\end{array}$ & $\begin{array}{l}\mathrm{H} / \\
\mathrm{cm}\end{array}$ & $\begin{array}{c}\mathrm{AEB} / \\
\mathrm{cm}\end{array}$ & $\begin{array}{l}\text { W1/ } \\
\text { gm }\end{array}$ & $\begin{array}{l}\text { W2/ } \\
\text { gm }\end{array}$ & $\begin{array}{c}\text { RWR } \\
\%\end{array}$ & E.S & N.B \\
\hline \multirow{5}{*}{ E.P } & without & 46 & 11.5 & 0.086 & 5 & 8.5 & 5 & 2.0 & 60 & - & - \\
\hline & 1Pz: & 24 & 6 & 0.167 & 2.5 & 4.5 & 5 & 0.90 & 82 & Yes & - \\
\hline & $2 \mathrm{Pz}:$ & 10 & 2.5 & 0.400 & 1.0 & 2.0 & 5 & 0.82 & 83 & Yes & - \\
\hline & 3 Pz: & 5 & 1.25 & 0.800 & 0.5 & 1.0 & 5 & 0.21 & 95 & Yes & - \\
\hline & $4 \mathrm{Pz}:$ & 7 & 1.75 & 0.572 & 1.0 & 1.2 & 5 & 0.27 & 94 & Yes & - \\
\hline \multirow{5}{*}{ P.U } & without & 299 & 74.75 & 0.013 & 6.0 & 15 & 5 & 4.5 & 10 & - & - \\
\hline & 1Pz: & 113 & 28.25 & 0.035 & 2.5 & 5.6 & 5 & 1.70 & 66 & Yes & - \\
\hline & $2 \mathrm{Pz}:$ & 94 & 23.5 & 0.042 & 2.0 & 4.7 & 5 & 1.41 & 71 & Yes & - \\
\hline & 3 Pz: & 87 & 21.75 & 0.045 & 2.0 & 4.3 & 5 & 1.31 & 73 & Yes & - \\
\hline & $4 \mathrm{Pz}:$ & 31 & 7.75 & 0.129 & 1.0 & 1.6 & 5 & 0.47 & 90 & Yes & - \\
\hline \multirow{5}{*}{ SBR } & without & 575 & 143.75 & 0.006 & 12.0 & 15.0 & 5 & 4.6 & 8 & - & - \\
\hline & 1Pz: & 126 & 31.5 & 0.031 & 3.5 & 3.3 & 5 & 1.01 & 79 & Yes & - \\
\hline & $2 \mathrm{Pz}:$ & 90 & 22.5 & 0.044 & 1.5 & 1.8 & 5 & 0.72 & 85 & Yes & - \\
\hline & $3 \mathrm{Pz}:$ & 75 & 18.75 & 0.053 & 1.5 & 2.0 & 5 & 0.60 & 88 & Yes & - \\
\hline & 4 Pz: & 84 & 21 & 0.047 & 1.5 & 2.2 & 5 & 0.67 & 86 & Yes & - \\
\hline \multirow{5}{*}{ A.S } & without & 498 & 124.5 & 0.008 & 10.0 & 15 & 6 & 5.1 & 15 & - & - \\
\hline & 1Pz: & 78 & 19.5 & 0.051 & 1.5 & 2.3 & 6 & 0.78 & 87 & Yes & - \\
\hline & $2 \mathrm{Pz}:$ & 57 & 14.25 & 0.070 & 1.5 & 1.5 & 6 & 0.56 & 90 & Yes & - \\
\hline & $3 \mathrm{Pz}:$ & 59 & 14.75 & 0.067 & 1.5 & 1.7 & 6 & 0.58 & 90 & Yes & - \\
\hline & $4 \mathrm{Pz}:$ & 53 & 13.25 & 0.075 & 1.0 & 0.6 & 6 & 0.53 & 91 & Yes & - \\
\hline \multirow{5}{*}{ PUF } & without & 306 & 76.5 & 0.013 & 12 & 15 & 4 & 3.60 & 10 & Yes & - \\
\hline & 1Pz: & 65 & 16.25 & 0.061 & 2.5 & 3.2 & 4 & 0.76 & 81 & Yes & - \\
\hline & $2 \mathrm{Pz}:$ & 36 & 9 & 0.111 & 1.5 & 1.7 & 4 & 0.43 & 89 & Yes & - \\
\hline & 3 Pz: & 38 & 9.5 & 0.105 & 1.5 & 1.9 & 4 & 0.45 & 88 & Yes & - \\
\hline & $4 \mathrm{Pz}:$ & 40 & 10 & 0.100 & 2.0 & 2.2 & 4 & 0.47 & 88 & Yes & - \\
\hline \multirow{5}{*}{ OD } & without & 362 & 90.5 & 0.011 & 8.0 & 15 & 6 & 4.3 & 28 & - & - \\
\hline & 1Pz: & 70 & 17.5 & 0.057 & 1.5 & 3.0 & 6 & 0.83 & 86 & Yes & - \\
\hline & 2 Pz: & 34 & 8.5 & 0.117 & 1.0 & 1.4 & 6 & 0.40 & 93 & Yes & - \\
\hline & 3 Pz: & 41 & 10.25 & 0.097 & 1.0 & 1.7 & 6 & 0.49 & 91 & Yes & - \\
\hline & $4 \mathrm{Pz}:$ & 45 & 11.25 & 0.089 & 1.0 & 1.8 & 6 & 0.53 & 91 & Yes & - \\
\hline \multirow{5}{*}{ W } & without & 588 & 147 & 0.006 & 12 & 15 & 5 & 4.8 & 4 & - & - \\
\hline & 1Pz: & 84 & 21 & 0.047 & 2.0 & 2.2 & 5 & 0.68 & 86 & Yes & - \\
\hline & $2 \mathrm{Pz}:$ & 54 & 13.5 & 0.074 & 1.0 & 1.5 & 5 & 0.44 & 91 & Yes & - \\
\hline & $3 \mathrm{Pz}:$ & 40 & 10 & 0.100 & 1.0 & 1.0 & 5 & 0.33 & 93 & Yes & - \\
\hline & $4 \mathrm{Pz}:$ & 37 & 9.25 & 0.108 & 1.0 & 0.9 & 5 & 0.31 & 93 & Yes & - \\
\hline \multirow{5}{*}{ PVA } & without & 372 & 93 & 0.011 & 13 & 15 & 6 & 4.5 & 25 & - & - \\
\hline & 1Pz: & 56 & 14 & 0.071 & 2.0 & 2.3 & 6 & 0.68 & 88 & Yes & - \\
\hline & 2 Pz: & 23 & 5.75 & 0.173 & 1.0 & 1.0 & 6 & 0.28 & 95 & Yes & - \\
\hline & $3 \mathrm{Pz}:$ & 21 & 5.25 & 0.190 & 1.0 & 0.9 & 6 & 0.25 & 95 & Yes & - \\
\hline & $4 \mathrm{Pz}:$ & 23 & 5.75 & 0.173 & 1.0 & 1.1 & 6 & 0.28 & 95 & Yes & - \\
\hline
\end{tabular}


Where:

$B . T=$ Burning Time (sec).

$I . I=$ Insulation Index $(\mathrm{sec} / \mathrm{mm})$.

$E . R=$ Erosion Rate $(\mathrm{mm} / \mathrm{sec})$.

$H=$ High flame $(\mathrm{cm})$.

A.E.B = Average extent of burning $(\mathrm{cm})$.

$W 1=$ Sample weight before burning (gm).

$W 2=$ Weight of the missing material (gm).

$R W R=$ Percentage of residual weight after combustion (\%).

E.S $=$ Self-Extinguishing.

$N . B=$ Non Burning.

$* P=$ only Cyclohexachlorotriphosphazene without Azo phenolic compounds.

$\mathrm{M}=$ cyclophosphazenes

$\mathrm{Pz}=$ Polyphosphazenes

\section{CONCLUSIONS}

Many constructional materials can be inhibited to fire extinguishing by using cyclophosphazene or polyphosphazene. The efficiency of phosphazenes as fire retarding materials can be developed by many order of magnitude throughout the improving of their chemical structure. The chemical structure of phosphazenes have been improved by increasing the number of halogen atoms with the molecule by linking with different azo moieties. It was noted that polyphosphazenes compounds were better than phosphazenes and azo compounds as fire retardant.

\section{REFERENCES}

Allcock, H.R.; Morozowich, N.L. (2012). Biodegradable Polyphosphazenes and their medical potential. Polum. Chem. 3, 578-590.

Allcock, H.R. (2006). Recent development in phosphazene materials science. Curr. Op. Solid st. M., 10, 231-240.

Arvind, S.A.; Dhiman, S.; Sandeep, A.; Kapoor, A. (2010). Synthesis of colon specific N,N-Bis-(2Chloroethyl) Aniline Polyphosphazene Copolymer conjugates. International J. Pharm. and Pharmaceut. Sci., 2(4), ISSN-0975-1491.

Camino, G.; Costa, L.; Cortemiglia, L.P. (1991). Stability of polymers. Polym. Degrad. Stabil., 33, 131

Cullis, F.C.; Hirschter, M.M. (1981). "The Combustion of Organic Polymers". Clarendon Press, Oxford, London, pp. 1-10.

Dittenber, B.D.; Gangarao, S.H.; Compos, Pt. (2012). Fire retardant materials. Appl. Sci. Manuf., 43, 1419

Khattab, A.F.; Abbas, M.F. (2015). Studies on storing and releasing the optical energy via azo polymers and its relation with the electrical conductivity. International J. Enhanced Research in Sci., Technol. Eng., 4(8), 1-10. August.

Schindler, W.D.; Hauser, P.J. (2004). "Chemical Finishing of Flame". Wood head, Cambridge, $1^{\text {st }}$ ed., $98 \mathrm{p}$.

Shumao, L.; Iie, R.; Hua, Y.; Taoa, Y.; Weixhong, Y. (2010). Some using of polymers. Polym. Int., 59, 242,

Wichman, I.S. (2003). Fire performance of a composite materials. Prog. Energy Combust. Sci., 29, 247. 